\title{
The Study of Based on RAPD Molecular Markers in Celery Cultivars Relationship
}

\section{Liu Pangyuan, Zhang Baohai, Zhao Hong, He Weiming, Wang Huijie}

National Engineering Research Center for Vegetables, Beijing, China

\section{Email address:}

liupangyuan@nercv.org (Liu Pangyuan), zhangbaohai@nercv.org (Zhang Baohai), zhaohong@nercv.org (Zhao Hong), heweiming@nercv.org (He Weiming),1416402647@qq.com (Wang Huijie)

\section{To cite this article:}

Liu Pangyuan, Zhang Baohai, Zhao Hong, He Weiming, Wang Huijie. The Study of Based on RAPD Molecular Markers in Celery Cultivars Relationship. Science Discovery. Vol. 5, No. 5, 2017, pp. 312-316. doi: 10.11648/j.sd.20170505.12

Received: March 21, 2017; Accepted: July 6, 2017; Published: August 4, 2017

\begin{abstract}
In this study, from forty different varieties of celery in different regions of China as test material, the scientific character of RAPD technology in the identification of celery cultivars was carried out in the verification and analysis technology. The results show that the RAPD can well distinguish the genetic relationship of celery varieties of far and near, is suitable for the analysis of celery cultivars relationship between simple and ideal DNA molecular marker technology. One of the thirteen bases of different length of primer RAPD technique in the analysis of celery cultivars relationship between definition and the number of bands have the obvious difference, method based identification as celery varieties with different genetic relationship.
\end{abstract}

Keywords: Celery, RAPD, Relationship

\section{芹菜品种资源亲缘关系的RAPD的研究}

刘庞源, 张宝海, 赵泓, 何伟明, 王慧杰

国家蔬菜工程技术研究中心, 北京, 中国

邮箱

liupangyuan@nercv.org（刘庞源）, zhangbaohai@nercv.org（张宝海），zhaohong@nercv.org（赵泓）, heweiming@nercv.org（何伟明）, 1416402647@qq.com（王慧杰）

摘要：本研究以来自中国不同地区四十种不同品种芹菜为试材，对RAPD技术在鉴定芹菜品种中的科学性进行了技术 上的验证与分析。结果表明通过RAPD技术能很好的区分芹菜品种间亲缘关系的远近, 是适用于分析芹菜品种亲缘关 系的简易与理想的DNA分子标记技术。其中十三个碱基数不同长度的引物的RAPD技术在芹菜品种亲缘关系分析中体 现出谱带清晰度以及数量等有明显区别, 以此为基础作为鉴定芹菜的不同品种的亲缘关系的方法。

关键词: 芹菜, RAPD, 亲缘关系

\section{1. 引言}

芹菜 (Apium graveolens L.) 是伞形花科二年生草本 植物, 原产地中海沿岸[1], 在中国已有2000多年的栽培历 史, 具有药用和保健功效, 深受种植者和消费者的喜爱。 目前中国南北各地均有种植, 芹菜种植面积逐年扩大, 可
以一年四季周年生产。目前中国国内种植的芹菜品种主要 是有地方品种和国外引进的杂交品种, 同名不同种及同种 不同名的现象较多, 且芹菜品种间形态特征差异不明显, 从植物学上很难区分, 易受环境和人为因素的影响, 再加 
上芹菜味二年生蔬菜, 生长期长, 因此进行芹菜优良性状 早期鉴定和遗传关系研究十分重要。

本研究提供了一种基于RAPD分子标记分析的芹菜品 种亲缘关系的分析方法。

\section{2. 材料与方法}

\section{1. 材料}

供试材料共 40 个, 分别引种于北京、天津、河北、西 安等地, 是当地的主栽品种, 请参见表 1 。

随机引物购自北京赛百盛基因技术有限公司, $2 \times$ EasyTaq PCR SuperMix 和DNA分子量标准Trans $5 \mathrm{~K}$ DNA Marker购于北京全式金生物技术有限公司。

表1 芹菜品种及其来源。

\begin{tabular}{|c|c|c|c|}
\hline 编号 & 保存编号 & 品种名称 & 详细来源 \\
\hline 1 & TC08001 & 斯地德西芹 & 河北农欢种业 \\
\hline 2 & TC08014 & 文图拉 & 天津TIANYU SEED \\
\hline 3 & TC08015 & 改良文图拉 & 北京嘉禾千秋农业技术研究所 \\
\hline 4 & TC08016 & 文图拉 & 北京市特种蔬菜种苗公司 \\
\hline 5 & TC08017 & 文图拉 & 北京美农东方科技发展有限公司 \\
\hline 6 & TC08018 & 文图拉 & 北京金丹隆种子有限公司 \\
\hline 7 & TC08019 & 聚星文图拉 & 北京聚萍兴利农业科技有限公司 \\
\hline 8 & TC08020 & 文图拉 & 北京绿金蓝种苗有限责任公司 \\
\hline 9 & TC08002 & 奥德斯西芹 & 河北农欢种业 \\
\hline 10 & TC08003 & 尤特 & 北京绿金蓝种苗有限责任公司 \\
\hline 11 & TC08004 & AX100 & 北京阿特拉斯种业有限公司 \\
\hline 12 & TC08005 & ATX200 & 北京阿特拉斯种业有限公司 \\
\hline 13 & TC08006 & Rising Sun & 天津科润蔬菜研究所 \\
\hline 14 & TC08007 & 皇菲 & 天津科润蔬菜研究所 \\
\hline 15 & TC08008 & 法国西芹 & 北京生光地公司 \\
\hline 16 & TC08009 & 皇后西芹 & 亘青（Tezier） \\
\hline 17 & TC08010 & 美琪 & 北京华耐种子有限公司 \\
\hline 18 & TC08011 & 美国百利 & 河北大禹种业有限公司 \\
\hline 19 & TC08012 & 精选加州王 & 北京市特种蔬菜种苗公司 \\
\hline 20 & TC08013 & CALIFORNIA EMPEROR & 北京市特种蔬菜种苗公司 \\
\hline 21 & TC08021 & 阿波罗 & 北京市特种蔬菜种苗公司 \\
\hline 22 & TC08022 & 玉皇 & RIJK ZWAAN \\
\hline 23 & TC08023 & 帝王 & RIJK ZWAAN \\
\hline 24 & TC08024 & 京芹1号 & 北京京研益农科技发展中心 \\
\hline 25 & TC08025 & 百利 & 河北邯䣋市裕康蔬菜种苗服务中心 \\
\hline 26 & TC08026 & 美国西芹 & 广州番禹丰顺种子经营部 \\
\hline 27 & TC08027 & 津锋 & 天津市兴科种子有限公司 \\
\hline 28 & TC08028 & 希望 & 河北邯䣋市永年裕康蔬菜种苗服务中心 \\
\hline 29 & TC08029 & 皇冠 & 法国 \\
\hline 30 & TC08030 & 名旌 & 北京绿金蓝种苗有限责任公司 \\
\hline 31 & TC08031 & 特选加州王 & 北京市特种蔬菜种苗公司 \\
\hline 32 & TC36001 & 四季西芹 & 天津科润蔬菜研究所 \\
\hline 33 & TC36002 & 百强西芹 & 天津科润蔬菜研究所 \\
\hline 34 & TC37001 & 本芹 & 甘肃张掖 \\
\hline 35 & TC37002 & 四季小香芹 & 北京市芳萱苑种子有限公司 \\
\hline 36 & TC37003 & 日本小香芹 & 北京市特种蔬菜种苗公司 \\
\hline 37 & TC37004 & 四季实芹 & 山东宁阳县华鲁种业有限公司 \\
\hline 38 & TC37005 & 马家沟芹菜 & 山东 \\
\hline 39 & TC38001 & 红芹 & 美毅 \\
\hline 40 & TC38002 & 紫芹菜 & 北京市特种蔬菜种苗公司 \\
\hline
\end{tabular}

\section{2. 取样及样品处理}

采用正常生长的芹菜品种, 每个品种 10 株, 每株 10 片新鲜幼叶放入冰盒, 置于 $-40^{\circ} \mathrm{C}$ 超低温冰箱中储存 $24 \mathrm{~h}$ 后 冷冻干燥成粉末状 (由 SCANVAC 公司生产的 CoolSafe ${ }^{\mathrm{TM}}-55-4$ 真空冻干机冻干后, 用手轻微揉碎), 将 其充分混匀;

\section{3. NA提取及其纯度和浓度监测}

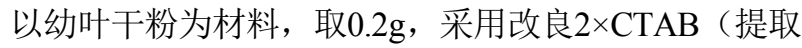
缓冲液）提取总DNA，使用 $0.8 \mathrm{w} \%$ 琼脂糖对所获基因组DNA 的纯度进行检测, 利用蛋白核酸定量测定仪检测DNA浓度。

\section{3. $1.2 \times$ CTAB的配制方法}

成分终浓度 
Tris-HCl缓冲液（pH8.0） 100 mmol/L,

EDTA-Na $\mathrm{Na}_{2}$ (EDTA二钠）（pH8.0）20mmol/L,

$\mathrm{NaCl} 1.4 \mathrm{~mol} / \mathrm{L}$,

CTAB2w\% (20g/L) ,

DNA提取前加入 $\beta$-統基乙醇 $2 \mathrm{ml} / \mathrm{L} （ 0.2 \mathrm{w} \%$ ）。

\subsubsection{DNA提取方法}

(1) 取 $6 \mathrm{ml}$ 预热到 $60^{\circ} \mathrm{C}$ 的 $2 \times \mathrm{CTAB}$ 溶液于装有 $0.2 \mathrm{~g}$ 叶 片粉末的离心管中, $60^{\circ} \mathrm{C}$ 水浴 30 分钟, 间或轻摇几次, 使 粉末和溶液混匀; (2) 取出离心管, 待冷却后加入 $6 \mathrm{ml}$ 氯仿-异戊醇（体积比24：1），置于摇床上30分钟充分混 匀; (3) $12000 \mathrm{rpm}$ 离心10分钟; (4) 将上清液转入新的 离心管中, 加入 2 倍体积预冷 $\left(-20^{\circ} \mathrm{C}\right)$ 无水乙醇, 混匀, 在 $-20^{\circ} \mathrm{C}$ 冰箱中放 30 分钟, 使核酸沉淀成絮状; (5) 室温 下12000 rpm离心10分钟; (6) 弃上清, DNA沉淀风干后,
溶解于 $800 \mu \mathrm{l}$ 去离子水中, 作为 $\mathrm{PCR}$ 模板 $\mathrm{DNA},-20^{\circ} \mathrm{C}$ 保 存备用;

使用 $0.8 \mathrm{w} \%$ 琼脂糖对所获基因组DNA的纯度进行检 测, 利用蛋白核酸定量测定仪检测DNA浓度。使用Thermo 公司NANODROP2000微量分光光度计直接测得DNA浓度, OD260/280 的比值在 1.8-2.0之间, OD260/230 的比值在 2.0-2.5之间, DNA浓度大于 $10 \mathrm{mg} / \mathrm{ml}$ 。

\section{4. 引物篮选和RAPD分析}

在对芹菜进行RAPD分析的PCR扩增体系优化一对 DNA模板量从5ng, 10ng, 20ng, 40ng, 60ng, 80ng, 100ng 进行优化, 最总选择20ng。然后, 选取40个（表1）芹菜 品种进行RAPD140个引物（见表2) 篮选。

从得到的DNA指纹图谱中, 笁选出 13 个扩增带清晰、 多态性明显、重复性好的引物，进行PCR扩增（见图1）。

表2 140 个随机引物编号及序列。

\begin{tabular}{|c|c|c|c|}
\hline SBSA & & & \\
\hline 01CAGGCCCTTC & 02TGCCGAGCTG & 03AGTCAGCCAC & 04AATCGGGCTG \\
\hline 05AGGGGTCTTG & 06GGTCCCTGAC & 07GAAACGGGTG & 08GTGACGTAGG \\
\hline 09GGGTAACGCC & 10GTGATCGCAG & 11CAATCGCCGT & 12TCGGCGATAG \\
\hline 13CAGCACCCAC & 14TCTGTGCTGG & 15TTCCGAACCC & 16AGCCAGCGAA \\
\hline 17GACCGCTTGT & 18AGGTGACCGT & 19CAAACGTCGG & 20GTTGCGATCC \\
\hline \multicolumn{4}{|l|}{ SBSB } \\
\hline 01GTTTCGCTCC & 02TGATCCCTGG & 03CATCCСCCТG & 04GGACTGGAGT \\
\hline 05TGCGCCCTTC & 06TGCTCTGCCC & 07GGTGACGCAG & 08GTCCACACGG \\
\hline 09TGGGGGACTC & 10CTGCTGGGAC & 11GTAGACCCGT & 12CCTTGACGCA \\
\hline 13TTCCCCCGCT & 14TCCGCTCTGG & 15GGAGGGTGTT & 16TTTGCCCGGA \\
\hline 17AGGGAACGAG & 18CCACAGCAGT & 19ACCCCCGAAG & 20GGACCCTTAC \\
\hline \multicolumn{4}{|l|}{ SBSC } \\
\hline 01TTCGAGCCAG & 02GTGAGGCGTC & 03GGGGGTCTTT & 04CCGCATCTAC \\
\hline 05GATGACCGCC & 06GAACGGACTC & 07GTCCCGACGA & 08TGGACCGGTG \\
\hline 09CTCACCGTCC & 10TGTCTGGGTG & 11AAAGCTGCGG & 12TGTCATCCCC \\
\hline 13AAGCCTCGTC & 14TGCGTGCTTG & 15GACGGATCAG & 16CACACTCCAG \\
\hline 17TTCCCCCCAG & 18TGAGTGGGTG & 19GTTGCCAGCC & 20ACTTCGCCAC \\
\hline \multicolumn{4}{|l|}{ SBSD } \\
\hline 01ACCGCGAAGG & 02GGACCCAACC & 03GTCGCCGTCA & 04TCTGGTGAGG \\
\hline 05TGAGCGGACA & 06ACCTGAACGG & 07TTGGCACGGG & 08GTGTGCCCCA \\
\hline 09CTCTGGAGAC & 10GGTCTACACC & 11AGCGCCATTG & 12CACCGTATCC \\
\hline 13GGGGTGACGA & 14CTTCCCCAAG & 15CATCCGTGCT & 16AGGGCGTAAG \\
\hline 17TTTCCCACGG & 18GAGAGCCAAC & 19CTGGGGACTT & 20ACCCGGTCAC \\
\hline \multicolumn{4}{|l|}{ SBSE } \\
\hline 01CCCAAGGTCC & 02GGTGCGGGAA & 03CCAGATGCAC & 04GTGACATGCC \\
\hline 05TCAGGGAGGT & 06AAGACCCCTC & 07AGATGCAGCC & 08TCACCACGGT \\
\hline 09CTTCACCCGA & 10CACCAGGTGA & 11GAGTCTCAGG & 12TTATCGCCCC \\
\hline 13CCCGATTCGG & 14TGCGGCTGAG & 15ACGCACAACC & 16GGTGACTGTG \\
\hline 17CTACTGCCGT & 18GGACTGCAGA & 19ACGGCGTATG & 20AACGGTGACC \\
\hline \multicolumn{4}{|l|}{ SBSM } \\
\hline 01GTTGGTGGCT & 02ACAACGCCTC & 03GGGGGATGAG & 04GGCGGTTGTC \\
\hline 05GGGAACGTGT & 06CTGGGCAACT & 07CCGTGACTCA & 08TCTGTTCCCC \\
\hline 09GTCTTGCGGA & 10TCTGGCGCAC & 11GTCCACTGTG & 12GGGACGTTGG \\
\hline 13GGTGGTCAAG & 14AGGGTCGTTC & 15GACCTACCAC & 16GTAACCAGCC \\
\hline 17TCAGTCCGGG & 18CACCATCCGT & 19CCTTCAGGCA & 20AGGTCTTGGG \\
\hline \multicolumn{4}{|l|}{ SBSN } \\
\hline 01CTCACGTTGG & 02ACCAGGGGCA & 03GGTACTCCCC & 04GACCGACCCA \\
\hline 05ACTGAACGCC & 06GAGACGCACA & 07CAGCCCAGAG & 08ACCTCAGCTC \\
\hline 09TGCCGGCTTG & 10ACAACTGGGG & 11TCGCCGCAAA & 12CACAGACACC \\
\hline 13AGCGTCACTC & 14TCGTGCGGGT & 15CAGCGACTGT & 16AAGCGACCTG \\
\hline 17CATTGGGGAG & 18GGTGAGGTCA & 19GTCCGTACTG & 20GGTGCTCCGT \\
\hline
\end{tabular}


40 个芹菜品种采用 13 个引物, 在Gene Amp PCR System 9700PCR扩增仪上进行DNA扩增。采用 $20 \mu \mathrm{L}$ 反应体系进行PCR 扩增, 反应体系总体积为 $20 \mu \mathrm{L}$, 含 $10 \mu \mathrm{L} 2 \times$ EasyTagPCRSuperMix (+dye) (北京全式金生物技术有限公司) $+2 \mu \mathrm{L} 5 \mathrm{mM}$ 引物+20ngDNA。

$\mathrm{PCR}$ 应程序为: $94^{\circ} \mathrm{C}$ 预变性 $5 \mathrm{~min} 30 \mathrm{~s}$; 接着 $94^{\circ} \mathrm{C}$ 变性 $1 \mathrm{~min} 30 \mathrm{~s}, 40^{\circ} \mathrm{C}$ 复性 $1 \mathrm{~min}, 72^{\circ} \mathrm{C}$ 延伸 $2 \mathrm{~min}, 40$ 个循环; 最后 $72^{\circ} \mathrm{C}$ 后延伸 $10 \mathrm{~min}$ 。

扩增产物用 $1.6 \mathrm{w} \%$ 、含 $1 \mathrm{v} \%$ Goldenview的琼脂糖凝胶电泳分离 $2-3 \mathrm{~h}$, 电压为 $6 \mathrm{~V} / \mathrm{cm}$; 在UV光下检测扩增结果并通过 凝胶成像系统 (GelDocXR ${ }^{+}$) 照相, 分别得到40种芹菜的随机扩增多态DNA标准图谱, 如其中SBSE20引物的扩增多态 DNA标准图谱（图1）。

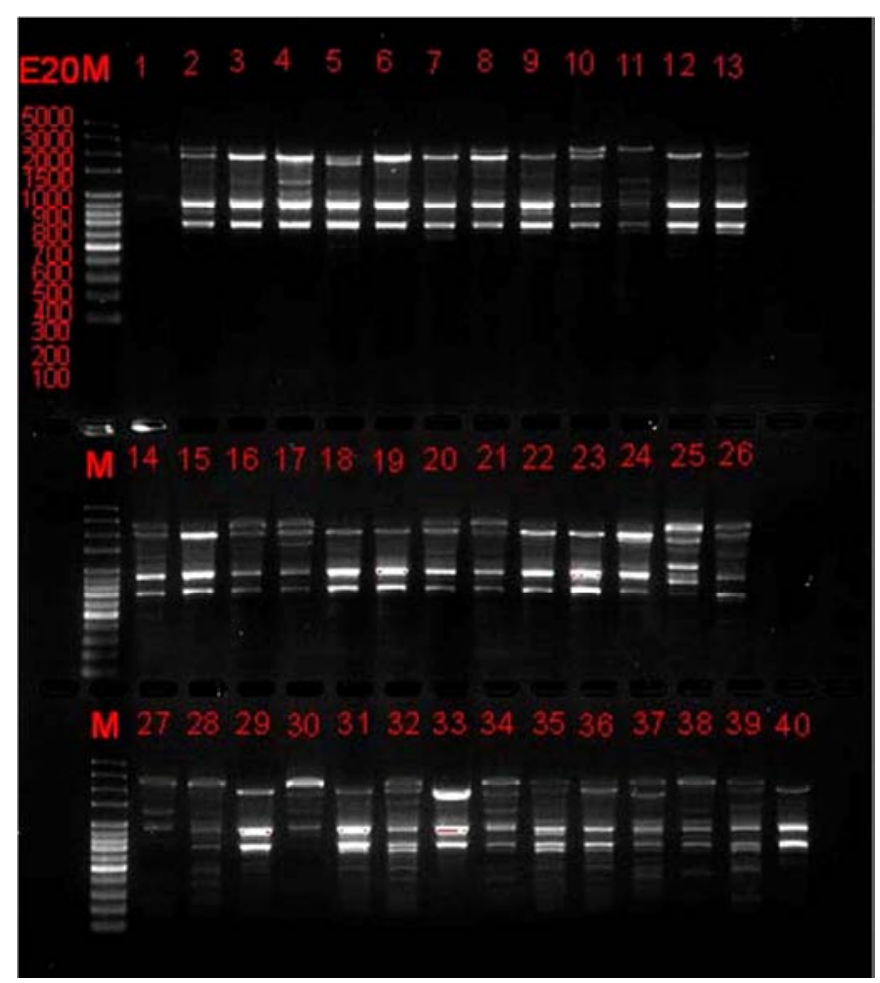

图1 40个芹菜品种采用引物SBS E20 (AACGGTGACC) 扩增的DNA标准图谱。

\section{5. 统计分析}

统计个样本扩增条带 (每条多条带视为一个等位基因, 有带赋值为 1 , 无带赋值为 0 ) 利用NTSYS软件对数据进行 分析以不加权成对算术平均法（UPGMA）对材料进行聚类分析作图（见图2）。

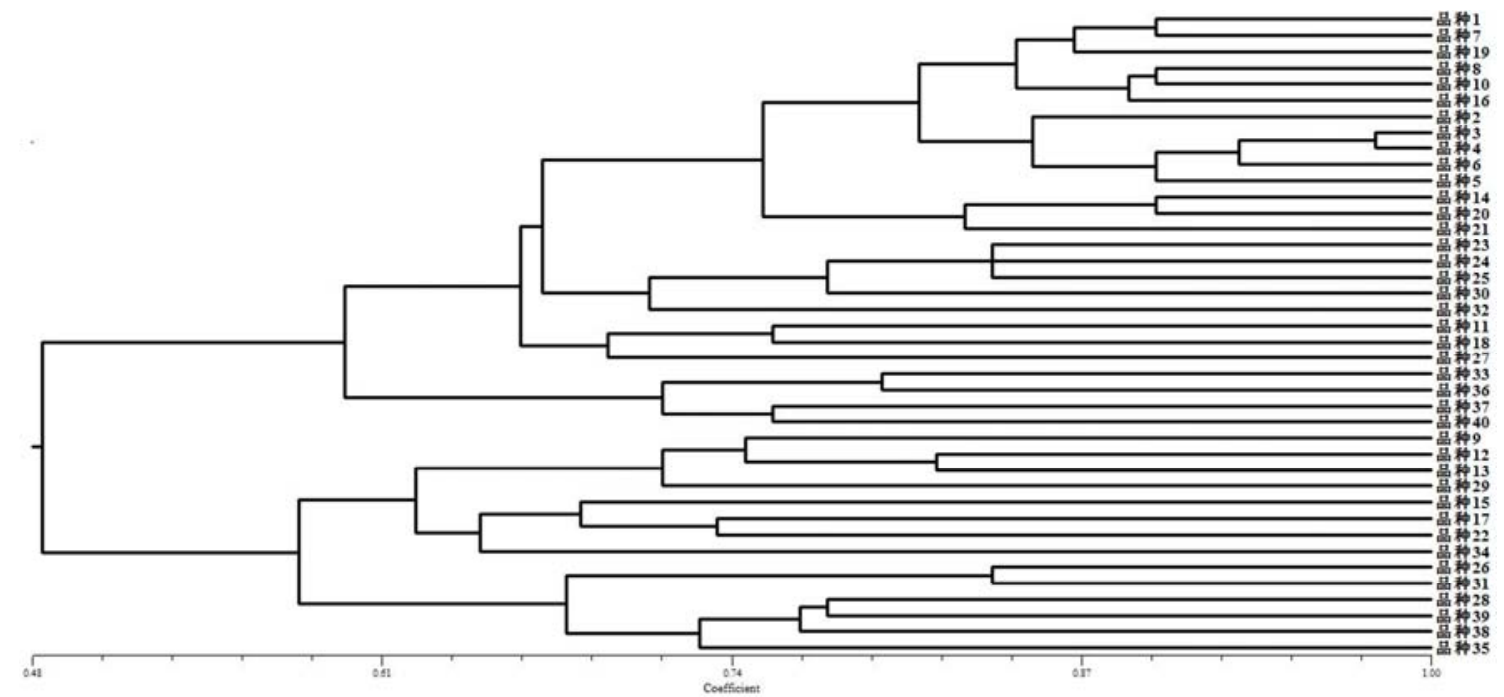

图2 40份芹菜品种聚类分析树状图。 


\section{3. 结果与分析}

每个引物扩增产生了一种特有RAPD指纹图谱, 从分 析得出的扩增的大部分图谱出现有, 如图 1 是用其中一个 SBS E20引物扩增的RAPD指纹图谱, 图中从左至右的图 谱编号分别为 M 和 1-40, 其中 M:DNA分子量标准, Trans5K DNA Marker Go149（北京全式金生物技术有限公司）; 1-40: 芹菜品种编号, 见表1。

对于供试芹菜品种的 5 个重复个体而言, 引物SBS E20 分别扩增产生相一致的DNA指纹图谱, 出现多态性, 40 种芹菜品种扩增图谱相互之间有明显相同和不同的条带, 从分子水平上说明, 能够判定芹菜品种亲缘关系的远近。

\section{4. 芹菜亲缘关系的鉴定}

根据分子标记数据获得的聚类图（图2），在相似系 数 0.61 为进行划分, 可将所有测试品种划分为 4 类。其中 第一类主要为西芹品种 22 份, 第二类品种好号为: 33,36 , 37,40 , 为本芹, 第三类为: $9 、 12 、 13 、 29 、 15 、 17$ 、 $22 、 34$ 属于西芹类型, 生长势强, 第四类为: $26 、 31 、 28$ 、 39、38、35, 属于西芹与本亲的中间类型。芹菜品种存在 地域差别, 随着交通便利发展和科技的进步, 地域影响正 在减小, 特别是北京地区, 由于地理位置的特殊性, 品种 起源比较复杂。

\section{5. 结论与讨论}

本试验聚类分析结果在遗传相似系数为 0.61 水平上, 将 40 个芹菜品种进行亲缘关系分析, 将 40 个品种分为四大 类, 第一类和第三类都属于西芹类型, 植株抱合紧实, 开 展度小, 叶柄实心, 叶肉厚, 口味较淡。但第一类和第三 类的区别是侧芽数普遍低于第一类的侧芽数, 有一定区别。 第二类为本芹类型, 植株松散, 开展度大, 叶肉较薄, 口 味浓。第四类为西芹与本芹的中间型, 叶柄比西芹叶柄窄, 叶柄较长, 实心。结果表明北方地区栽培的主要芹菜资源 亲缘关系较近, 聚类分析结果与地域来源和形态特征有一 定对应关系。本研究的分析表明大部分芹菜资源集中到第 一类, 说明芹菜资源亲缘关系较近, 也说明我国栽培的芹 菜资源遗传背景比较狭窄。另外, 中国本芹与西芹在形态 上具有极大的区别, 但个别西芹品种归类到本芹类中，但 比较分散, 与某些本芹的亲缘关系较近。另外也有个别本 芹品种归属到西芹类别中, 也有可能是同名异物造成的结
果或是经过长期的栽培极有可能与其他种杂交出现不同 方向的进化，同时也证实本芹与西芹具有较近的亲缘关系。

\section{参考文献}

[1] Determination of genetic diversity among Saccharina germplasm using ISSR and RAPD markers Cuiju Cui; Yan Li; Yanling Liu; Xiaojie Li; Shiju Luo; Zhuan... Comptes rendus - Biologies 2016-06-15.

[2] Genetic diversity study amongst Cymbopogon species from NE-India using RAPD and ISSR markers Baruah; B. Gogoi; K. Das; N. M. Ahmed; D. K. Sarmah; M. Lal. Industrial Crops \& Products 2016-10-13.

[3] 白苟种质资源的DNA分子标记研究徐攀; 梁卫青; 周洁; 张宏建; 胡轶娟中华中医药学刊2017-04-10。

[4] An N, Guo H B, KEW D. Genetic Variation in Rhizome Lotus (Nelumbo nucifera Gaertn. ssp. nucifera) Germplasms from China Assessed by RAPD Markers[J]. Agricultural Sciences in China, 2009, 8(1):31 39.

[5] Yang RW, Zhou YH, Ding C B, eta. 1 Biologia Plantarum, Relationships among Leymus species assessed by RAPD markers[J]. Biologia Plantarum, 2008, 52(2):237 241.

[6] Wang Y J, Lu JN. The research on RAPD genetic markers of grape seed lessness gene[J]. Journal of Northwest A \& F University Na.t Sc.i Ed., 1996, 5(24):10 20.

[7] Williams JG K, Kubelik A R, Liva k. J, et a.l DNA polymorphisms amplified by arbitrary primers are useful as genetic markers[J]. Nucl Acids Res, 1990, 18:6531 6535.

[8] Lin KH, Lai Y C, Li H C. Genetic variation and its relationship to root weight in the Sweet potato as revealed by RAPD analysis[J]. Scientia Horti culturae, 2009, 120(1):2 7.

[9] Cai Y L, Cao D W, Zhao G F. Studies on genetic variation in cherry germplasm using RAPD analysis[J]. Scientia Horticulturae, 2007, 111(3):248 254.

[10] 马艳芝, 张玉星. 梨种质资源遗传多样性研究中的RAPD 技术引物篎选[J]. 中国农学通报, 2009, 25 (11)：30 33。

[11] 高道侠, 文海涛, 林励, 等. 江西酸橙不同栽培变种RAPD 分析[J]. 安徽农业科学, 2009, 37 （8）：3457 3459。

[12] 李莉, 彭建营, 白瑞霞. 中国束属植物亲缘关系的RAPD 分析[J]. 园艺学报，2009，36（4）：475 480。 